(C)2008 IEEE. Personal use of this material is permitted. However, permission to reprint/republish this material for advertising or promotional purposes or for creating new collective works for resale or redistribution to servers or lists, or to reuse any copyrighted component of this work in other works must be obtained from the IEEE. 


\title{
Optimal Beamforming for Non-Regenerative MIMO Relays with Direct Link
}

\author{
Yue Rong, Member, IEEE, and Feifei Gao, Member, IEEE
}

\begin{abstract}
In this letter, we generalize the existing works on the design of the optimal relay amplifying matrix for nonregenerative multiple-input multiple-output (MIMO) relay communication systems by including the direct source-destination link. We show that for most commonly used objective functions, the optimal relay amplifying matrix has a general beamforming structure, that is, the relay first sets beams to the direction of the source-relay channel, then conducts a linear precoding, and finally beamforms towards the direction of the relay-destination channel.
\end{abstract}

Index Terms-MIMO relay, linear non-regenerative relay, direct link.

\section{INTRODUCTION}

$\mathbf{R}$ ECENTLY, non-regenerative multiple-input multipleoutput (MIMO) relay communications attract much research interest. For a MIMO relay system, there are two independent links between the source and the destination nodes: the source-relay-destination link and the direct sourcedestination link. Many works studied the optimal relay amplifying matrix for the source-relay-destination channel. In [1], [2], the optimal relay amplifying matrix which maximizes the mutual information (MI) between source and destination was derived. In [3], [4], the relay amplifying matrix was designed to minimize the mean-squared error (MSE) of the signal waveform estimation at the destination. An optimal relay amplifying matrix based on the maximum signal-to-noise ratio (SNR) criterion was developed in [4]. All these works did not consider the direct source-destination link.

In practice, the direct source-destination link provides valuable spatial diversity to the MIMO relay system and should not be ignored. Obviously, the relay amplifying matrix designed for the source-relay-destination link only [1]-[4] is not optimal when the direct link is included. It was conjectured in [1] that with the direct link, the left coordinate of the optimal relay amplifying matrix is given by the right singular matrix of the relay-destination channel. In [2], the structure of the relay amplifying matrix that maximizes the source-destination MI in the presence of the direct link was derived. However, the structure of such relay amplifying matrix is suboptimal, since it does not consider the structure of the transmission power constraint at the relay node.

In this letter, we investigate the optimal relay amplifying matrix for non-regenerative MIMO relay communication systems with the presence of the direct source-destination link.

Manuscript received September 8, 2009. The associate editor coordinating the review of this letter and approving it for publication was P. Demestichas.

Y. Rong is with the Department of Electrical and Computer Engineering, Curtin University of Technology, Bentley, WA 6102, Australia (e-mail: y.rong@curtin.edu.au).

F. Gao is with the School of Engineering and Science, Jacobs University, Bremen, 28759, Germany (e-mail: feifeigao@ieee.org).

Digital Object Identifier 10.1109/LCOMM.2009.12.091810
We prove the conjecture in [1] and show that for most commonly used objective functions, the optimal relay amplifying matrix has a general beamforming structure. First, the relay performs receive beamforming using the Hermitian transpose of the left singular matrix of the source-relay channel. Then the relay conducts a linear precoding operation. Finally, a transmit beamforming is performed by the relay using the right singular matrix of the relay-destination channel. In contrast to MIMO relay systems with only the source-relay-destination link, a closed-form solution of the optimal linear precoding matrix can not be obtained. Nonetheless, one can always resort to numerical ways to optimize the linear precoding matrix. Numerical example demonstrates the effectiveness of our algorithm.

\section{SySTEM MODEL}

We consider a three-node MIMO communication system where the source node transmits information to the destination node with the aid of one relay node. The source, relay, and destination nodes are equipped with $N_{s}, N_{r}$, and $N_{d}$ antennas, respectively. Due to its merit of simplicity, a non-regenerative strategy is applied at the relay node to amplify and forward the received signal. The signal vector received at the destination node over two consecutive time slots is

$$
\begin{aligned}
\mathbf{y}(t) & \triangleq\left[\begin{array}{c}
\mathbf{y}_{d}(t+1) \\
\mathbf{y}_{d}(t)
\end{array}\right] \\
& =\left[\begin{array}{c}
\mathbf{H}_{r d} \mathbf{F} \mathbf{H}_{s r} \\
\mathbf{H}_{s d}
\end{array}\right] \mathbf{G s}(t)+\left[\begin{array}{c}
\mathbf{H}_{r d} \mathbf{F} \mathbf{v}_{r}(t)+\mathbf{v}_{d}(t+1) \\
\mathbf{v}_{d}(t)
\end{array}\right](1)
\end{aligned}
$$

where $\mathbf{y}_{d}(t+1)$ and $\mathbf{y}_{d}(t)$ are $N_{d} \times 1$ signal vectors received at the destination through the source-relay-destination link and the direct source-destination link, respectively, $\mathbf{H}_{s d}, \mathbf{H}_{r d}$, $\mathbf{H}_{s r}$ are the channel matrices for the source-destination, relaydestination, and source-relay links with dimension $N_{d} \times N_{s}$, $N_{d} \times N_{r}, N_{r} \times N_{s}$, respectively, $\mathbf{s}(t)$ is the $N_{s} \times 1$ source signal vector, $\mathbf{F}$ is the $N_{r} \times N_{r}$ relay amplifying matrix, $\mathbf{G}$ is the $N_{s} \times N_{s}$ source precoding matrix, $\mathbf{v}_{r}(t)$ is the $N_{r} \times 1$ noise vector at the relay, $\mathbf{v}_{d}(t+1)$ and $\mathbf{v}_{d}(t)$ are the $N_{d} \times 1$ noise vectors at the destination at time $t+1$ and $t$, respectively.

We assume that the source signal vector satisfies $\mathrm{E}\left[\mathbf{s}(t)(\mathbf{s}(t))^{H}\right]=\mathbf{I}_{N_{s}}$ and all noises are independent and identically distributed (i.i.d.) additive white Gaussian noise (AWGN) with zero mean and unit variance. Here E[.] stands for the statistical expectation, $\mathbf{I}_{n}$ is an $n \times n$ identity matrix, and $(\cdot)^{H}$ denotes the Hermitian transpose. We also assume that the relay and destination nodes know all the channel state information (CSI). However, the CSI is unavailable to the source node. Thus, the source chooses $\mathbf{G}=\sqrt{P_{s} / N_{s}} \mathbf{I}_{N_{s}}$, where $P_{s}>0$ is the transmission power available at the source node. 
When a linear receiver is used at the destination node, the estimated signal waveform is given by

$$
\hat{\mathbf{s}}(t)=\mathbf{W}^{H} \mathbf{y}(t)
$$

where $\mathbf{W}$ is a $2 N_{d} \times N_{s}$ weight matrix. The receiver weight matrix which minimizes the signal waveform estimation error is the Wiener filter given by [5]

$$
\mathbf{W}=\left(\overline{\mathbf{H}} \overline{\mathbf{H}}^{H}+\overline{\mathbf{C}}\right)^{-1} \overline{\mathbf{H}}
$$

where

$$
\overline{\mathbf{H}} \triangleq \sqrt{\rho}\left[\begin{array}{c}
\mathbf{H}_{r d} \mathbf{F} \mathbf{H}_{s r} \\
\mathbf{H}_{s d}
\end{array}\right], \overline{\mathbf{C}} \triangleq\left[\begin{array}{ll}
\mathbf{H}_{r d} \mathbf{F F} \mathbf{F}^{H} \mathbf{H}_{r d}^{H}+\mathbf{I}_{N_{d}} & \mathbf{0}_{N_{d} \times N_{d}} \\
\mathbf{0}_{N_{d} \times N_{d}} & \mathbf{I}_{N_{d}}
\end{array}\right] .
$$

Here $\mathbf{0}_{m \times n}$ denotes an $m \times n$ matrix with all zeros entries, $(\cdot)^{-1}$ denotes the matrix inversion, and $\rho \triangleq P_{s} / N_{s}$. Using (1)-(3), the MSE matrix $\mathbf{E}$ of the signal waveform estimation is given by

$$
\begin{aligned}
\mathbf{E}= & \mathrm{E}\left[(\hat{\mathbf{s}}(t)-\mathbf{s}(t))(\hat{\mathbf{s}}(t)-\mathbf{s}(t))^{H}\right] \\
= & {\left[\mathbf{I}_{N_{s}}+\rho \mathbf{H}_{s d}^{H} \mathbf{H}_{s d}+\rho \mathbf{H}_{s r}^{H} \mathbf{F}^{H} \mathbf{H}_{r d}^{H}\right.} \\
& \left.\times\left(\mathbf{H}_{r d} \mathbf{F} \mathbf{F}^{H} \mathbf{H}_{r d}^{H}+\mathbf{I}_{N_{d}}\right)^{-1} \mathbf{H}_{r d} \mathbf{F} \mathbf{H}_{s r}\right]^{-1} .
\end{aligned}
$$

\section{Optimal RELAY BEAMFORMing}

In this section, we derive the structure of the optimal relay amplifying matrix. Most commonly used objective functions in MIMO system design are closely linked to the MSE matrix $\mathbf{E}$ [6]. For example, the negative MI objective is MI $=\log _{2}|\mathbf{E}|$, where $|\cdot|$ denotes the matrix determinant. The MSE objective is given as MSE $=\operatorname{tr}(\mathbf{E})$, where $\operatorname{tr}(\cdot)$ denotes the trace of a matrix. In the sequel, we use $q(\mathbf{E})$ as a unified notation for the objective function. It is worth noting that any practical objective function should be an increasing function of $\mathbf{E}$, i.e., if $\mathbf{E}_{1} \preceq \mathbf{E}_{2}$, then $q\left(\mathbf{E}_{1}\right) \leq q\left(\mathbf{E}_{2}\right)$, where $\mathbf{E}_{1} \preceq \mathbf{E}_{2}$ means that $\mathbf{E}_{2}-\mathbf{E}_{1}$ is a positive semidefinite matrix.

The relay amplifying matrix optimization problem is written as

$$
\begin{array}{ll}
\min _{\mathbf{F}, \mathbf{E}} & q(\mathbf{E}) \\
\text { s.t. } & \operatorname{tr}\left(\mathbf{F}\left(\rho \mathbf{H}_{s r} \mathbf{H}_{s r}^{H}+\mathbf{I}_{N_{r}}\right) \mathbf{F}^{H}\right) \leq P_{r}
\end{array}
$$

where (6) is the power constraint at the relay node, and $P_{r}>0$ is the power budget available at the relay. Let us introduce the following singular value decomposition

$$
\mathbf{H}_{s r}=\mathbf{U}_{s} \boldsymbol{\Lambda}_{s} \mathbf{V}_{s}^{H}, \quad \mathbf{H}_{r d}=\mathbf{U}_{r} \boldsymbol{\Lambda}_{r} \mathbf{V}_{r}^{H}
$$

where $\Lambda_{s}$ and $\Lambda_{r}$ are $R_{s} \times R_{s}$ and $R_{r} \times R_{r}$ square diagonal matrices (i.e., zero singularvalues are excluded). Here $R_{s} \triangleq$ $\operatorname{rank}\left(\mathbf{H}_{s r}\right), R_{r} \triangleq \operatorname{rank}\left(\mathbf{H}_{r d}\right), \operatorname{rank}(\cdot)$ denotes the rank of a matrix. The following theorem states the structure of the optimal $\mathbf{F}$.

Theorem 1: If $q\left(\mathbf{E}_{1}\right) \leq q\left(\mathbf{E}_{2}\right)$ for $\mathbf{E}_{1} \preceq \mathbf{E}_{2}$, then the optimal $\mathbf{F}$ is given by

$$
\mathbf{F}=\mathbf{V}_{r} \mathbf{A} \mathbf{U}_{s}^{H} .
$$

Proof: Without loss of generality, we write $\mathbf{F}$ as

$$
\mathbf{F}=\left[\begin{array}{ll}
\mathbf{V}_{r} & \mathbf{V}_{r}^{\perp}
\end{array}\right]\left[\begin{array}{cc}
\mathbf{A} & \mathbf{B} \\
\mathbf{C} & \mathbf{D}
\end{array}\right]\left[\begin{array}{c}
\mathbf{U}_{s}^{H} \\
\left(\mathbf{U}_{s}^{\perp}\right)^{H}
\end{array}\right]
$$

where $\mathbf{V}_{r}^{\perp}\left(\mathbf{V}_{r}^{\perp}\right)^{H}=\mathbf{I}_{N_{r}}-\mathbf{V}_{r} \mathbf{V}_{r}^{H}, \mathbf{U}_{s}^{\perp}\left(\mathbf{U}_{s}^{\perp}\right)^{H}=\mathbf{I}_{N_{r}}-$ $\mathbf{U}_{s} \mathbf{U}_{s}^{H}$, such that $\overline{\mathbf{V}}_{r} \triangleq\left[\begin{array}{ll}\mathbf{V}_{r} & \mathbf{V}_{r}^{\perp}\end{array}\right]$ and $\overline{\mathbf{U}}_{s} \triangleq\left[\begin{array}{ll}\mathbf{U}_{s} & \mathbf{U}_{s}^{\perp}\end{array}\right]$ are unitary matrices, $\mathbf{A}, \mathbf{B}, \mathbf{C}, \mathbf{D}$ are arbitrary matrices with dimensions of $R_{r} \times R_{s}, R_{r} \times\left(N_{r}-R_{s}\right),\left(N_{r}-R_{r}\right) \times R_{s}$, $\left(N_{r}-R_{r}\right) \times\left(N_{r}-R_{s}\right)$, respectively. Applying the matrix inversion lemma $(\mathbf{T}+\mathbf{X Y Z})^{-1}=\mathbf{T}^{-1}-\mathbf{T}^{-1} \mathbf{X}\left(\mathbf{Z} \mathbf{T}^{-1} \mathbf{X}+\right.$ $\left.\mathbf{Y}^{-1}\right)^{-1} \mathbf{Z T}^{-1}$, (4) can be written as

$$
\begin{aligned}
\mathbf{E} & =\left[\mathbf{I}_{N_{s}}+\rho \mathbf{H}_{s d}^{H} \mathbf{H}_{s d}+\rho \mathbf{H}_{s r}^{H}\right. \\
& \left.\times\left[\mathbf{I}_{N_{r}}-\left(\mathbf{I}_{N_{r}}+\mathbf{F}^{H} \mathbf{H}_{r d}^{H} \mathbf{H}_{r d} \mathbf{F}\right)^{-1}\right] \mathbf{H}_{s r}\right]^{-1} .
\end{aligned}
$$

Substituting (7) and (9) into (10) we have

$$
\mathbf{F}^{H} \mathbf{H}_{r d}^{H} \mathbf{H}_{r d} \mathbf{F}=\overline{\mathbf{U}}_{s}\left[\begin{array}{cc}
\mathbf{A}^{H} \boldsymbol{\Lambda}_{r}^{2} \mathbf{A} & \mathbf{A}^{H} \boldsymbol{\Lambda}_{r}^{2} \mathbf{B} \\
\mathbf{B}^{H} \boldsymbol{\Lambda}_{r}^{2} \mathbf{A} & \mathbf{B}^{H} \boldsymbol{\Lambda}_{r}^{2} \mathbf{B}
\end{array}\right] \overline{\mathbf{U}}_{s}^{H} .
$$

From (10) and (11) we see that the objective function (5) does not depend on C and D. Substituting (11) back into (10) we have

$$
\begin{aligned}
& \mathbf{E}=\left[\mathbf{I}_{N_{s}}+\rho \mathbf{H}_{s d}^{H} \mathbf{H}_{s d}+\rho \mathbf{V}_{s} \boldsymbol{\Lambda}_{s}\left(\mathbf{I}_{R_{s}}-\mathbf{U}_{s}^{H} \overline{\mathbf{U}}_{s}\right.\right. \\
& \left.\left.\times\left[\begin{array}{ll}
\mathbf{A}^{H} \boldsymbol{\Lambda}_{r}^{2} \mathbf{A}+\mathbf{I}_{R_{s}} & \mathbf{A}^{H} \boldsymbol{\Lambda}_{r}^{2} \mathbf{B} \\
\mathbf{B}^{H} \boldsymbol{\Lambda}_{r}^{2} \mathbf{A} & \mathbf{B}^{H} \boldsymbol{\Lambda}_{r}^{2} \mathbf{B}+\mathbf{I}_{N_{r}-R_{s}}
\end{array}\right]^{-1} \overline{\mathbf{U}}_{s}^{H} \mathbf{U}_{s}\right) \boldsymbol{\Lambda}_{s} \mathbf{V}_{s}^{H}\right]^{-1} \\
& =\left[\mathbf{I}_{N_{s}}+\rho \mathbf{H}_{s d}^{H} \mathbf{H}_{s d}+\rho \mathbf{H}_{s r}^{H} \mathbf{H}_{s r}-\rho \mathbf{V}_{s} \boldsymbol{\Lambda}_{s}\right. \\
& \left.\times\left(\mathbf{A}^{H} \boldsymbol{\Lambda}_{r}^{2} \mathbf{A}+\mathbf{I}_{R_{s}}-\mathbf{M}\right)^{-1} \boldsymbol{\Lambda}_{s} \mathbf{V}_{s}^{H}\right]^{-1}
\end{aligned}
$$

where we applied the matrix inversion lemma for partitioned matrix to obtain the second equation, and $\mathbf{M} \triangleq$ $\mathbf{A}^{H} \boldsymbol{\Lambda}_{r}^{2} \mathbf{B}\left(\mathbf{B}^{H} \boldsymbol{\Lambda}_{r}^{2} \mathbf{B}+\mathbf{I}_{N_{r}-R_{s}}\right)^{-1} \mathbf{B}^{H} \boldsymbol{\Lambda}_{r}^{2} \mathbf{A}$. Since $q(\mathbf{E})$ is increasing with respect to $\mathbf{E}$, in order to minimize $q(\mathbf{E}), \mathbf{E}$ should be minimized. It is well-known that for two positive definite matrices $\mathbf{A}$ and $\mathbf{B}$, if $\mathbf{A} \succ \mathbf{B}$, then $\mathbf{A}^{-1} \prec \mathbf{B}^{-1}$. Thus, from (12), $\mathbf{E}$ is minimized if $\mathbf{M}=\mathbf{0}_{R_{s} \times R_{s}}$, which holds if $\mathbf{B}=\mathbf{0}_{R_{r} \times\left(N_{r}-R_{s}\right)}$.

Now we look at the constraint (6). The power consumed by the relay node can be rewritten as

$$
\begin{aligned}
& \operatorname{tr}\left(\mathbf{F}\left(\rho \mathbf{H}_{s r} \mathbf{H}_{s r}^{H}+\mathbf{I}_{N_{r}}\right) \mathbf{F}^{H}\right)=\operatorname{tr}\left(\mathbf{A}\left(\rho \boldsymbol{\Lambda}_{s}^{2}+\mathbf{I}_{R_{s}}\right) \mathbf{A}^{H}\right. \\
& \left.+\mathbf{B} \mathbf{B}^{H}+\mathbf{C}\left(\rho \boldsymbol{\Lambda}_{s}^{2}+\mathbf{I}_{R_{s}}\right) \mathbf{C}^{H}+\mathbf{D D}^{H}\right) .
\end{aligned}
$$

Obviously, $\mathbf{B}=\mathbf{0}_{R_{r} \times\left(N_{r}-R_{s}\right)}, \mathbf{C}=\mathbf{0}_{\left(N_{r}-R_{r}\right) \times R_{s}}$, and $\mathbf{D}=$ $\mathbf{0}_{\left(N_{r}-R_{r}\right) \times\left(N_{r}-R_{s}\right)}$ minimize the power consumption. Thus we have $\mathbf{F}=\mathbf{V}_{r} \mathbf{A} \mathbf{U}_{s}^{H}$.

Theorem 1 shows that the optimal relay amplifying matrix can be viewed as a general form of beamforming. The relay first performs receive beamforming using the Hermitian transpose of the left singular matrix of the source-relay channel $\mathbf{U}_{s}^{H}$. Then the relay conducts a linear precoding operation using A. Finally, a transmit beamforming is performed by the relay using the right singular matrix of the relay-destination channel $\mathbf{V}_{r}$. It has been shown in [1]-[4] that without the direct link, the optimal relay amplifying matrix is

$$
\mathbf{F}=\mathbf{V}_{r_{1}} \mathbf{A} \mathbf{U}_{s_{1}}^{H}
$$

where $\mathbf{V}_{r_{1}}$ and $\mathbf{U}_{s_{1}}$ contain $L \triangleq \min \left(R_{s}, R_{r}\right)$ columns of $\mathbf{V}_{r}$ and $\mathbf{U}_{s}$ associated with the largest $L$ singular values, respectively, and $\mathbf{A}$ is an $L \times L$ diagonal matrix. Obviously, Theorem 1 includes [1]-[4] as special cases. 


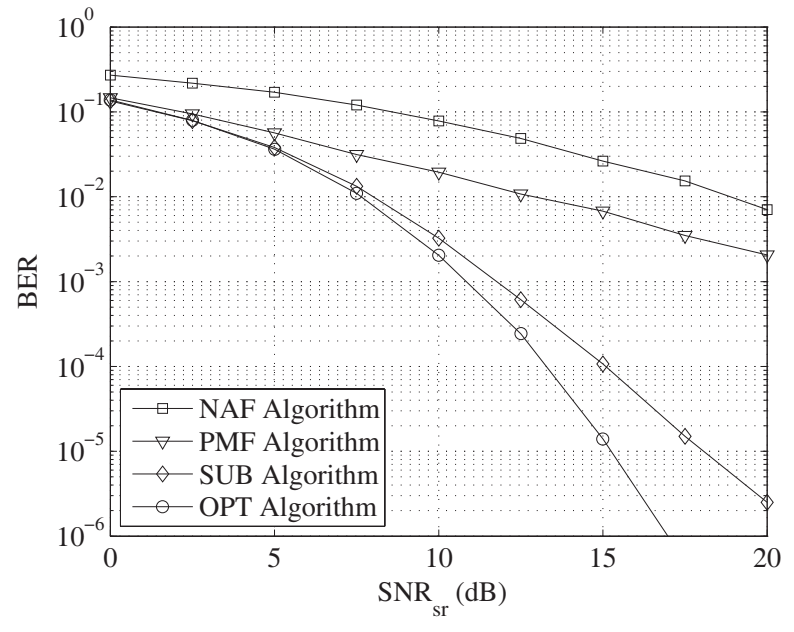

Fig. 1. BER versus $\mathrm{SNR}_{\mathrm{sr}} \cdot N_{s}=N_{d}=2, N_{r}=6, \mathrm{SNR}_{\mathrm{rd}}=20 \mathrm{~dB}$, $\mathrm{SNR}_{\mathrm{sd}}=\mathrm{SNR}_{\mathrm{sr}}-10 \mathrm{~dB}$.

The remaining task is to optimize A. From (12) and (13), we can write the optimization problem as

$$
\begin{array}{ll}
\min _{\mathbf{A}} & q\left(\left[\mathbf{I}_{N_{s}}+\rho \mathbf{H}_{s d}^{H} \mathbf{H}_{s d}+\rho \mathbf{H}_{s r}^{H} \mathbf{H}_{s r}\right.\right. \\
& \left.\left.-\rho \mathbf{V}_{s} \boldsymbol{\Lambda}_{s}\left(\mathbf{A}^{H} \boldsymbol{\Lambda}_{r}^{2} \mathbf{A}+\mathbf{I}_{R_{s}}\right)^{-1} \boldsymbol{\Lambda}_{s} \mathbf{V}_{s}^{H}\right]^{-1}\right) \\
\text { s.t. } & \operatorname{tr}\left(\mathbf{A}\left(\rho \boldsymbol{\Lambda}_{s}^{2}+\mathbf{I}_{R_{s}}\right) \mathbf{A}^{H}\right) \leq P_{r} .
\end{array}
$$

Both problem (5)-(6) and problem (15)-(16) have matrix optimization variable. However, in the former problem, the optimization variable $\mathbf{F}$ is an $N_{r} \times N_{r}$ matrix. While in the latter problem, the dimension of the optimization variable $\mathbf{A}$ is only $R_{r} \times R_{s}$. Since $R_{r} \leq N_{r}$ and $R_{s} \leq N_{r}$, the dimensional reduction from $N_{r}^{2}$ to $R_{s} R_{r}$ can be quite significant. Note that if $\mathbf{H}_{s d} \neq \mathbf{0}_{N_{d} \times N_{s}}$, there is no specific structure for the optimal $\mathbf{A}$ for general $\mathbf{H}_{s d}$ and $\mathbf{A}$ should be designed as a general matrix.

Now let us look at a specific example of $N_{s}=1$ and there is no constraint on $N_{r}$ and $N_{d}$. The MSE matrix is a scalar in this case and is given by $E=\left[1+\rho\left(\mathbf{h}_{s d}^{H} \mathbf{h}_{s d}+\right.\right.$ $\left.\left.\mathbf{h}_{s r}^{H} \mathbf{h}_{s r}-\lambda_{s}^{2}\left(\mathbf{a}^{H} \boldsymbol{\Lambda}_{r}^{2} \mathbf{a}+1\right)^{-1}\right)\right]^{-1}$, where for clarity we rewrite the corresponding matrices terms in (15) into vectors and scalars. In this example, the optimization problem (15)-(16) is equivalent to

$$
\max _{\mathbf{a}} \mathbf{a}^{H} \boldsymbol{\Lambda}_{r}^{2} \mathbf{a} \quad \text { s.t. }\left(\rho \lambda_{s}^{2}+1\right) \mathbf{a}^{H} \mathbf{a} \leq P_{r} .
$$

The solution to problem (17) is $\mathbf{a}=$ $\left[\sqrt{P_{r} /\left(\rho \lambda_{s}^{2}+1\right)}, \mathbf{0}_{1 \times\left(R_{r}-1\right)}\right]^{T}$. Since in this example $\mathbf{u}_{s}=\mathbf{h}_{s r} /\left\|\mathbf{h}_{s r}\right\|$, the optimal relay amplifying matrix is $\mathbf{F}=\frac{\sqrt{P_{r} /\left(\rho \lambda_{s}^{2}+1\right)}}{\left\|\mathbf{h}_{s r}\right\|} \mathbf{v}_{1}^{H} \mathbf{h}_{s r}$, where $\left\|\mathbf{h}_{s r}\right\|=\sqrt{\mathbf{h}_{s r}^{H} \mathbf{h}_{s r}}$ and $\mathbf{v}_{1}$ is the column vector of $\mathbf{V}_{r}$ associated with the largest singular value. We find that for $N_{s}=1, \mathbf{F}$ is a rank-one matrix. This indicates that the relay first forms a "beam" towards the direction of the source-relay channel, and then points the "beam" to the strongest direction of the relay-destination channel. Interestingly, in this case the direct link does not affect $\mathbf{F}$ for any $N_{r}$ and $N_{d}$.

Unfortunately, for $N_{s} \geq 2$, the problem (15)-(16) does not have a closed-form solution for general $\mathbf{H}_{s d}$. We should resort to numerical methods, such as the projected gradient method [7] to solve (15)-(16).

\section{NUMERICAL EXAMPLE}

We simulate a MIMO relay system with $N_{s}=N_{d}=2$ and $N_{r}=6$. All channel matrices have Gaussian entries with zero-mean and variances $\sigma_{s}^{2} / N_{s}, \sigma_{r}^{2} / N_{r}, \sigma_{d}^{2} / N_{s}$ for $\mathbf{H}_{s r}, \mathbf{H}_{r d}$, and $\mathbf{H}_{s d}$, respectively. Consequently, the SNRs are defined as $\mathrm{SNR}_{\mathrm{sr}} \triangleq \sigma_{s}^{2} P_{s} / N_{s}, \mathrm{SNR}_{\mathrm{rd}} \triangleq \sigma_{r}^{2} P_{r} / N_{r}, \mathrm{SNR}_{\mathrm{sd}} \triangleq \sigma_{d}^{2} P_{s} / N_{s}$ for the source-relay, relay-destination, and source-destination links, respectively. We simulate a scenario where the distance between the relay and destination nodes is fixed, while the source-relay distance (and thus also the source-destination distance) are varying. We set $\mathrm{SNR}_{\mathrm{rd}}=20 \mathrm{~dB}, \mathrm{SNR}_{\mathrm{sd}}=$ $\mathrm{SNR}_{\mathrm{sr}}-10 \mathrm{~dB}$. All simulation results are averaged over 1000 independent channel realizations.

We compare the performance of the optimal (OPT) algorithm in (8) with the naive amplify-and-forward (NAF) algorithm where $\mathbf{F}=\sqrt{P_{r} / \operatorname{tr}\left(\rho \mathbf{H}_{s r} \mathbf{H}_{s r}^{H}+\mathbf{I}_{N_{r}}\right)} \mathbf{I}_{N_{r}}$, the pseudo match-and-forward (PMF) algorithm where $\mathbf{F}=$ $\sqrt{P_{r} / \operatorname{tr}\left(\left(\mathbf{H}_{s r} \mathbf{H}_{r d}\right)^{H}\left(\rho \mathbf{H}_{s r} \mathbf{H}_{s r}^{H}+\mathbf{I}_{N_{r}}\right) \mathbf{H}_{s r} \mathbf{H}_{r d}\right)}\left(\mathbf{H}_{s r} \mathbf{H}_{r d}\right)^{H}$, and the suboptimal (SUB) algorithm (14) which optimizes only the source-relay-destination link. For both the OPT and the SUB algorithms, we chose MSE $\operatorname{tr}(\mathbf{E})$ as the objective function. For the OPT algorithm, the projected gradient method is applied to optimize $\mathbf{A}$ in (15)-(16).

Fig. 1 shows the performance of four algorithms in terms of bit-error-rate (BER) versus SNR $_{\text {sr }}$ using the QPSK constellation. It can be seen that the NAF algorithm has the worst performance, since it does not exploit the information on $\mathbf{H}_{r d}$. The OPT algorithm outperforms all competing algorithms in the whole $\mathrm{SNR}_{\mathrm{sr}}$ range. In fact, it achieves a much higher diversity order than the other algorithms.

\section{CONCLusion}

In this letter, we have derived the general structure of the optimal relay amplifying matrix for non-regenerative MIMO relay systems in the presence of the direct source-destination link. Such relay amplifying matrix is optimal for most commonly used objectives.

\section{REFERENCES}

[1] X. Tang and Y. Hua, "Optimal design of non-regenerative MIMO wireless relays," IEEE Trans. Wireless Commun., vol. 6, pp. 1398-1407, Apr. 2007.

[2] O. Muñoz-Medina, J. Vidal, and A. Agustín, "Linear transceiver design in nonregenerative relays with channel state information," IEEE Trans. Signal Process., vol. 55, pp. 2593-2604, June 2007.

[3] W. Guan and H. Luo, "Joint MMSE transceiver design in nonregenerative MIMO relay systems," IEEE Commun. Lett., vol. 12, pp. 517-519, July 2008.

[4] A. S. Behbahani, R. Merched, and A. M. Eltawil, "Optimizations of a MIMO relay network," IEEE Trans. Signal Process., vol. 56, pp. 50625073, Oct. 2008.

[5] S. M. Kay, Fundamentals of Statistical Signal Processing: Estimation Theory. Englewood Cilffs, NJ: Prentice Hall, 1993.

[6] D. P. Palomar, J. M. Cioffi, and M. A. Lagunas, "Joint Tx-Rx beamforming design for multicarrier MIMO channels: a unified framework for convex optimization,' IEEE Trans. Signal Process., vol. 51, pp. 23812401, Sep. 2003.

[7] D. P. Bertsekas, Nonlinear Programming, 2nd edition. Belmont, MA: Athena Scientific, 1995. 